\title{
A Rare Case of Congenital Coronary Artery Fistula Evaluated by Cardiac MRI
}

\section{Disawal A}

Government Medical College \& Hospital, Hanuman nagar, Nagpur, India

\begin{abstract}
In a patient with coronary artery fistula extending from anterior aortic sinus to posterosuperior wall of right atrium, Magnetic Resonance Imaging was able to accurately demonstrate dilatation of the involved coronary artery, the tortuous nature of the dilated fistula, blood flow within the fistula and its communication with right atrium. Coronary artery fistulas are among the rare anomalies of coronary arteries. Role of angiography is well established in identification and characterization of these anomalies, however their accurate course and termination is often not defined. We demonstrate role of cardiovascular MRI in non- invasively diagnosing and characterizing the course of these anomalous coronary branches. Here we report a rare case of coronary artery fistula, symptomatic due to hemodynamically significant coronary steal phenomenon. Magnetic Resonance Imaging revealed abnormal dilated tortuous channel extending from anterior aortic sinus and posterosuperior wall of right atrium suggestive of right coronary artery fistula. Large opening of the fistula was repaired with SFD patch and opening of the fistula in right atrium was closed directly with prolene.
\end{abstract}

Keywords: Magnetic Resonance Imaging, Coronary artery fistula, Right atrium

\section{Introduction}

A coronary artery fistula involves a sizeable communication between a coronary artery, bypassing the myocardial capillary bed and entering either a chamber of the heart (coronary-cameral fistula) or any segment of the systemic or pulmonary circulation (coronary arteriovenous fistula). The pathophysiology of these lesions is identical

Correspondence to: Dr. Amit Disawal Address: 1157, C.A. Road, $2^{\text {nd }}$ Floor, Above Dena Bank, Bhavsar Chowk, Gandhibagh, Nagpur, 440032.

E-mail: amitdisawal@yahoo.com and they are often collectively termed coronary arterial-venous fistulae (CAVFs). Percutaneous closure offers a safe and effective way for the management of symptomatic patients. CAFs are rare cardiac anomalies but can give rise to a variety of symptoms because of their hemodynamic consequences or complications.

\section{Case Report}

A 35 years old female presented with complaints of dyspnoea and chest pain (NYHA grade II) since 2 months. Clinical examination revealed blood pressure of 124/76 mm Hg, clear lung fields and grade 
4/6 continous murmur along the left sternal border. Radiograph chest revealed cardiomegaly with increased pulmonary vascular markings (Fig.1).

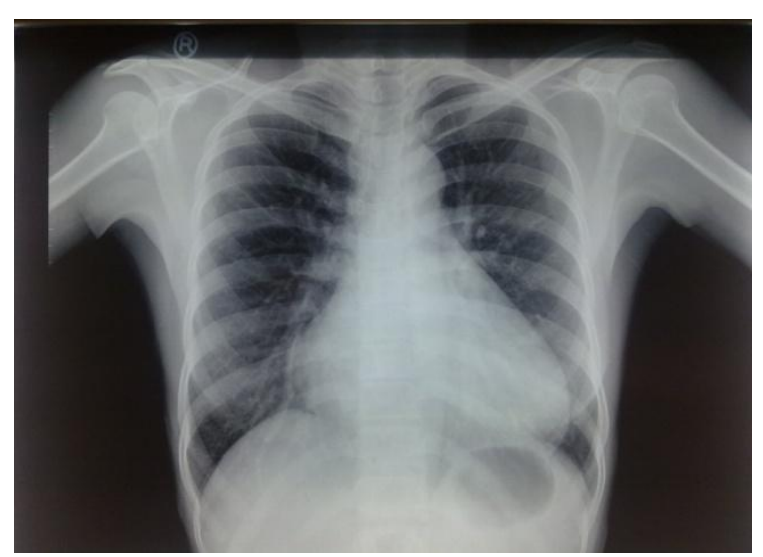

Fig. 1: Radiograph chest revealed biventricular cardiomegaly with increased pulmonary vascular markings

Two-Dimentional \& Doppler

Echocardiography (2D Echo) study was performed. Para-sternal long \& short axis and apical views revealed continuous flow in the right atrial cavity via a fistulous tract most probably from right coronary artery which was hugely dilated at its origin (Fig. $2 \mathrm{a}$ and $\mathrm{b})$.

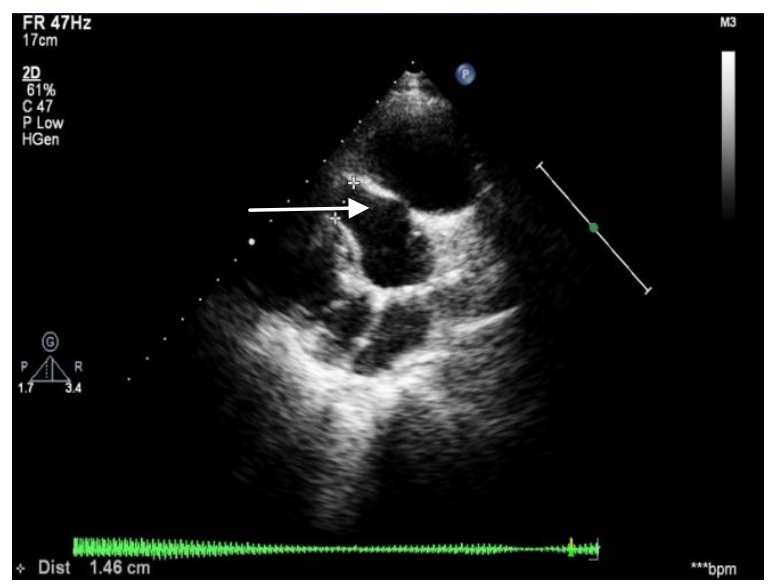

Fig. 2a: Echocardiography image showing origin of fistula from right anterior coronary sinus.

Cardiac MRI revealed mild dilatation of anterior aortic sinus with well defined dilated

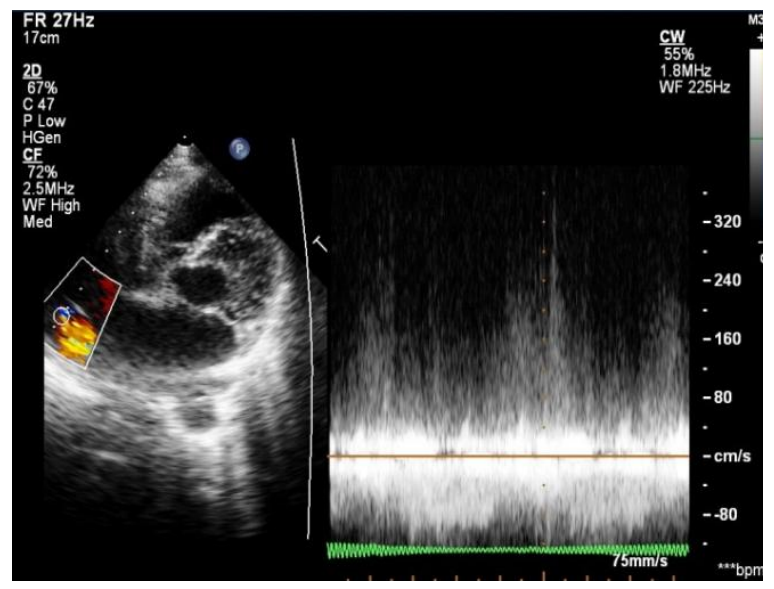

Fig. 2b: Echocardiography image showing continuous flow in right atrium

tortuous channels of approximate diameter $1.5 \mathrm{~cm}$ noted arising from the anterior aortic sinus coursing anteriorly between the right ventricular outflow tract and right atrial appendage reaching upto the roof of right atrium and opening in relation to anterosuperior part. The channel was found to be patent throughout its course causing left to right shunting of blood (Fig. 3 a, b, c, d)

\section{Treatment}

Cardiac catheterization is usually performed in order to confirm anatomy and plan surgical treatment. Our MR images matched the angiography findings so closely that surgery was performed without catheterization in this patient.

Median sternotomy aorto-bicaval cannulation followed by followed by cold blood cardioplegia was performed.

Large opening of the Coronary-RA fistula was repaired with Sauvage Filamentous Dacron (SFD) patch and opening of the fistula in RA was closed directly with prolene. Two drains were kept and the wound was closed in layers. 

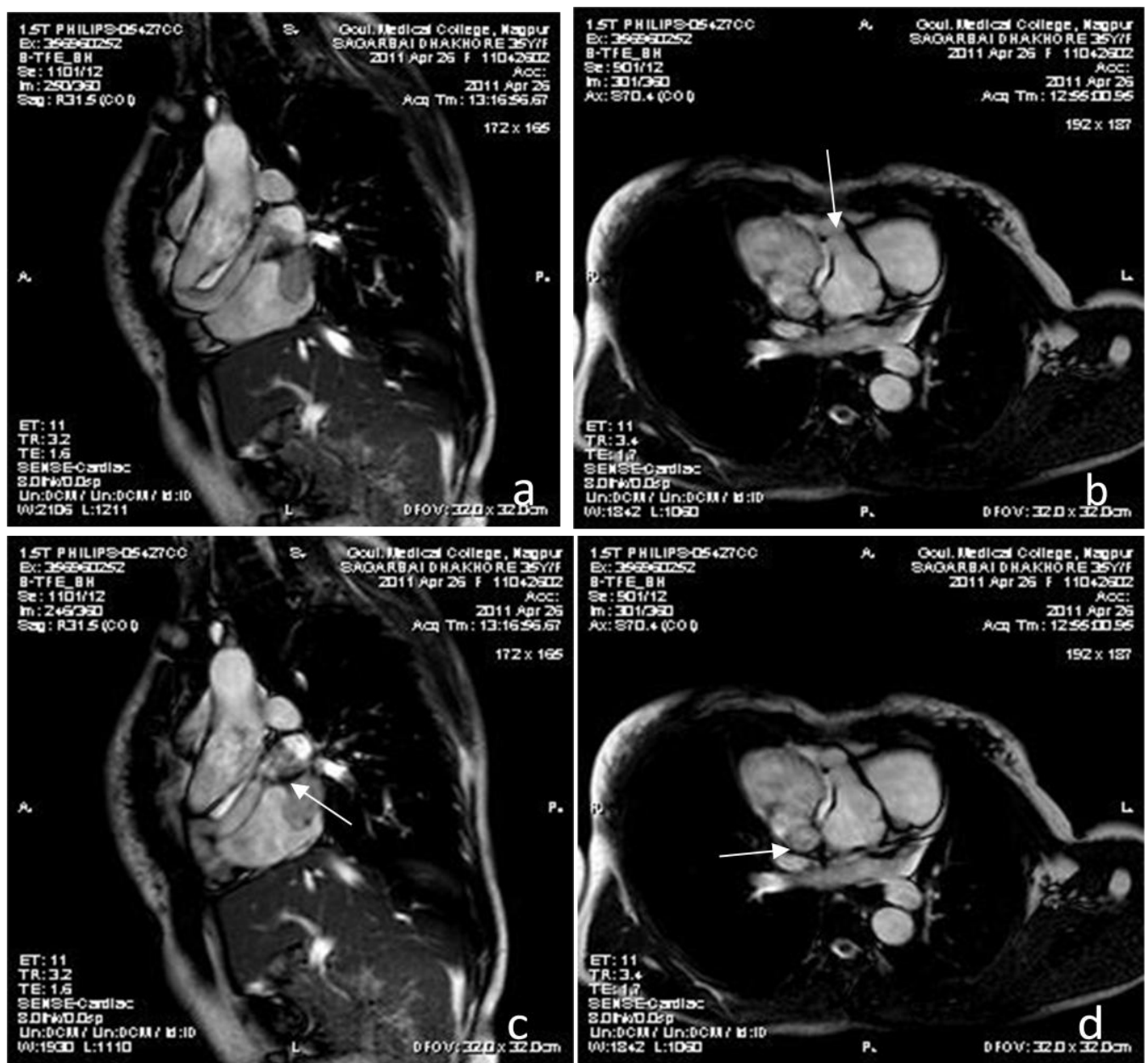

Fig. 3 a) BTFE sagittal image showing coronary fistula arising from right anterior coronary sinus and extending between root of aorta and right atrium. b) BTFE axial image showing coronary fistula arising from right anterior coronary sinus. c) BTFE sagittal oblique image showing opening of fistula in right atrium. d) BTFE axial image showing opening of fistula into posterosuperior wall right atrium.

\section{Discussion}

Coronary artery fistula (CAF) is an anomalous connection between a coronary artery and a major vessel or cardiac chamber. These fistulae bypass the myocardial capillary bed and enter either a chamber of the heart (coronary-cameral fistula) or any segment of the systemic or pulmonary circulation (coronary arteriovenous fistula).

These abnormal connections can lead to a severe hemodynamic compromise, which can affect myocardial perfusion. ${ }^{1}$
Coronary fistulas were first described in 1865, but their diagnostic triad was not described until 1978. ${ }^{2}$ Haller and Little characterized these lesions by the presence of an abnormal continuous murmur ${ }^{1}$ similar to that of a patent ductus arteriosus (PDA), a left-to-right shunt, and a large coronary artery with evidence of fistula on an angiogram. ${ }^{2}$ They can arise as a result of coronary atherosclerosis, Takayasu's arteritis, or trauma. ${ }^{3-5}$ Complications associated with coronary fistulas are heart failure, myocardial ischemia, infective endocarditis, arrhythmias, and rupture. ${ }^{5}$ Many coronary 
fistulas, however, are asymptomatic and are found incidentally.

Previous population studies using angiography revealed the incidence of coronary artery fistula as approximately $0.3 \% .^{6}$ Congenital coronary fistulas are the most common type of hemodynamically significant congenital coronary anomaly. A significant congenital coronary anomaly includes origin of the left coronary artery from the pulmonary artery, congenital coronary atresia, and origin of the left coronary artery from the right sinus of Valsalva, with subsequent passage between the great vessels. ${ }^{2}$ In the present case, the patient had no history of vasculitis, coronary artery disease, or trauma, indicating most likely a congenital coronary fistula with delayed presentation during middle age.

Clinical symptoms associated with coronary artery fistulas are variable and largely depend on the magnitude of the left-to-right shunt. ${ }^{3}$ In a large series of 51 patients with coronary fistulas, angina pectoris occurred in $57 \%$ of cases $^{7}$ and was often present in the absence of underlying coronary artery disease. Ischemia is thought to be caused by a phenomenon known as coronary steal, whereby blood flow is shifted away from the distal coronary vascular bed. ${ }^{4,8}$ Patients can also present with fatigue or dyspnea on exertion, either as an anginal equivalent, or as a manifestation of congestive heart failure. Presence of a large left-to-right shunt from the coronary fistula can lead to pulmonary hypertension and right ventricular failure., ${ }^{4,8}$

Other potential complications include infective endocarditis, ischemia or infarction-related arrhythmias, and coronary rupture. ${ }^{3,4}$ The patient described here had a continuous murmur in the precordium, which might have been caused by the coronary fistula. A murmur associated with a coronary fistula and left-to-right flow might become systolic after the development of pulmonary hypertension. It is, therefore, likely that mild pulmonary hypertension in this patient could be attributable to the coronary fistula. Furthermore, the patient presented with exertional dyspnea and arrhythmia, which was most likely a manifestation of coronary steal away from the distal right coronary artery vasculature, resulting in inferior ischemia and ischemic ventricular arrhythmia. Without closure of the fistula, this patient could be at risk of sudden cardiac death.

Although various techniques are available for the diagnosis of coronary fistulas, most are discovered using coronary angiography. Cardiac catheterization is usually performed in order to confirm anatomy and plan surgical treatment. Our MR images matched the angiography findings so closely, that surgery was performed without catheterization in this last patient.

A variety of approaches can be used to manage coronary artery fistulas. Clinical symptoms of ischemia, such as exertional angina or dyspnea, are the primary indication for closure of a fistula. Indeed, controversy exists regarding the management of fistulas in the absence of associated clinical symptoms. 4

Although fistulas sometimes close spontaneously, this is a rare occurrence. Surgical vessel ligation was first reported in 1947 and because it demonstrated good longterm safety and efficacy, this technique remained the most frequently chosen 
treatment option until the first successful percutaneous closure was reported in 1983. ${ }^{1,}$ 13, 14 Catheter-based closure methods have since become the preferred treatment option, if technically feasible. This technique can be performed with detachable balloons, stainless steel coils, controlled-release coils, controlled-release PDA coils and the Amplatzer $^{\circledR}$ PDA occluder (AGA Medical Corporation, Golden Valley, MN). ${ }^{15}$ The most frequent complication associated with catheter-based closure is embolization of the occlusion device, and in one study this complication occurred in $7(\sim 17 \%)$ out of 40 patients. $^{15}$ Catheter based closure has become established as the preferred method of fistula closure in experienced centers.

\section{Conclusion}

Here, we describe a patient with a hemodynamically significant coronary artery fistula to the right atrium, which was a congenital defect. The patient was affected by significant clinical symptoms, which were resolved following surgical closure of the fistula. Although coronary artery fistulas are rare anomalies, they should always be considered in a diagnostic work-up because they can result in cardiac symptoms and associated complications. With the advent of novel diagnostic techniques like cardiac MRI. Cardiac MRI not only diagnoses but also extent of coronary fistulas can be established more easily and also obviate the need of conventional coronary angiography.

\section{References}

1. Haller JA Jr, Little JA. Diagnosis and surgical correction of congenital coronary artery-coronary sinus fistula. Circulation 1963;27:939-942.
2. Levin DC. Hemodynamically significant primary anomalies of the coronary arteries: angiographic aspects. Circulation 1978;58:25-34.

3. Balanescu S. Coronary artery fistulas: clinical consequences and methods of closure. A literature review. Ital Heart J 2001;2:669-676.

4. Gowda RM. Coronary artery fistulas: clinical and therapeutic considerations. Int J Cardiol 2006; 107: 7-10.

5. Sapin P. Coronary artery fistula: an abnormality affecting all age groups. Medicine (Baltimore) 1990;69:101113.

6. Yamanaka O, Hobbs RE. Coronary artery anomalies in 126,595 patients undergoing coronary arteriography. Cathet Cardiovasc Diagn 1990;21:2840.

7. Said SA, van der Werf T. Dutch survey of coronary artery fistulas in adults: congenital solitary fistulas.mInt $\mathbf{J}$ Cardiol 2006;106:323-332.

8. Umana E. Myocardial ischemia secondary to a large coronarypulmonary fistula-a case report. Angiology 2002;53:353-357.

9. Lin FC. Multiplane transesophageal echocardiography in the diagnosis of congenital coronary artery fistula. Am Heart J 1995;130:1236-1244.

10. Goswami NJ, Zabalgoitia M. Localization of a coronary artery fistula 
using contrast transesophageal echocardiography. J Am Soc Echocardiogr 2002;15:839-840.

11. Schmid M. Visualization of coronary artery anomalies by contrast-enhanced multi-detector row spiral computed tomography. Int J Cardiol 2005; 111:430-435.

12. Rathi VK. Coronary artery fistula; noninvasive diagnosis by cardiovascular magnetic resonance imaging. $\mathrm{J}$ Cardiovasc Magn Reson 2005;7:723735 .

13. Kamiya H. Surgical treatment of congenital coronary artery fistulas: 27 years' experience and a review of the literature. J Card Surg 2002;17:173177.
14. Reidy JF. Transcatheter occlusion of coronary to bronchial anastomosis by detachable balloon combined with coronary angioplasty at same procedure. Br Heart J 1983;49:284287.

15. Qureshi SA, Tynan M. Catheter closure of coronary artery fistulas. J Interv Cardiol 2001;14:299-307. 